\title{
Publisher's Note: Multilayer tube impedance and external radiation [Phys. Rev. ST Accel. Beams 11, 084001 (2008)]
}

M. Ivanyan, E. Laziev, V. Tsakanov, A. Vardanyan, S. Heifets, and A. Tsakanian (Received 28 August 2008; published 9 September 2008)

DOI: $10.1103 /$ PhysRevSTAB.11.099901

PACS numbers: 41.60.- m, 41.75.Ht, 42.82.Et, 99.10.Fg

This paper was published online on 13 August 2008 without additional author corrections incorporated into this article. The paper has been corrected as of 27 August 2008. 